\title{
Estudo sobre a integridade superficial do aço ABNT 4140 após a operação de torneamento
}

\author{
Study on the surface integrity of AISI 4140 \\ steel after turning
}

\author{
Augusto Moura Martins ${ }^{1}$, Poliana Santos Souza ${ }^{1}$, \\ Anderson Júnior dos Santos ${ }^{1}$, Alexandre Mendes Abrão ${ }^{1}$, \\ Berend Denkena $^{2}$, Bernd Breidenstein ${ }^{2}$, Kolja Meyer ${ }^{2}$
}

\footnotetext{
${ }^{1}$ Programa de Pós-Graduação em Engenharia Mecânica, Universidade Federal de Minas Gerais, Av. Antônio Carlos, 6627, CEP: 31270-901, Pampulha, Belo Horizonte, Minas Gerais, Brasil.

${ }^{2}$ Institute of Production Engineering and Machine Tools, Leibniz Universität Hannover, An der Universität 2, 30823

Garbsen, Hanôver, Germany.

e-mail: augusto.mmartins2@gmail.com, polianassouza1@gmail.com,ndersonsantos@hotmail.com,

abrao@demec.ufmg.br,denkena@ifw.uni-hannover.de, breidenstein@ifw.uni-hannover.de, meyer@ifw.uni-hannover.de
}

\section{RESUMO}

Os componentes de equipamentos estão muitas vezes sujeitos a esforços cíclicos, os quais são capazes de provocar falhas, mesmo apenas quando deformações elásticas estão envolvidas. Os processos de fabricação são capazes de afetar a rugosidade e induzir tensões residuais de diferentes intensidades e profundidades, podendo influenciar o desempenho e a vida em serviço de peças manufaturadas. Neste trabalho, foi avaliada a influência dos parâmetros de torneamento (velocidade de corte, avanço e profundidade de usinagem) sobre a rugosidade, tensões residuais e vida em fadiga do aço ABNT 4140 temperado e revenido (40 HRC). A maior influência sobre a rugosidade foi devido à variação do avanço, que gerou maiores rugosidades para valores mais altos de avanço. Observou-se também que os parâmetros de usinagem foram capazes de alterar o perfil das tensões residuais, induzindo tensões de tração e de maior intensidade à medida que a velocidade de corte foi elevada e de tração em profundidades maiores para a combinação de avanço mais elevado e profundidade de usinagem mais baixa. A vida em fadiga do material foi avaliada por meio do teste por flexão rotativa, o qual acusou influência combinada do avanço com a profundidade de usinagem. Para menores avanços, o aumento da profundidade prolongou a vida em fadiga, já para avanços mais elevados o comportamento foi oposto. A vida em fadiga mais longa foi observada após o torneamento sob velocidade de corte e profundidade de usinagem elevadas e aliadas ao menor avanço.

Palavras-chave: Torneamento; qualidade superficial; rugosidade; vida em fadiga; aço ABNT 4140.

\begin{abstract}
Machine components are usually subjected to cyclic loads, which may lead to failure, even when the component is subjected to elastic deformations only. Manufacturing processes are capable of affecting roughness and inducing residual stresses of distinct levels and depths, being able to affect the component performance and lifespan. This work investigates the influence of turning parameters (cutting speed, feed and depth of cut) on surface roughness, residual stress and fatigue life of hardened AISI 4140 steel (40 HRC). Feed is the most influential parameter affecting surface roughness. It was noticed that the cutting parameters did affect the residual stress profile, i.e., tensile residual stresses of higher intensity were induced when higher cutting speeds were employed and reaching deeper layers when higher feed and lower depth of cut values were used. The fatigue life was evaluated under rotating bending tests, which indicated a combined influence of feed and depth of cut. At lower feeds, the increase of depth of cut resulted in longer lives, however, an opposite behavior was noted at higher feeds. Longer fatigue lives were observed after turning at higher cutting speeds and depths of cut combined with lower feeds.
\end{abstract}

Keywords: Turning; surface quality; roughness; fatigue life; AISI 4140 steel. 


\section{INTRODUÇÃO}

As operações de usinagem com ferramentas de geometria definidas são capazes de afetar a integridade superficial dos componentes produzidos e, consequentemente, sua resistência à fadiga 0. JAVIDI et al. 0 afirmam que os parâmetros de usinagem, tais como velocidade de corte, avanço e raio de ponta da ferramenta são conhecidos por afetar a integridade superficial da peça, alterando a rugosidade e as tensões residuais.

Ao considerar a influência dos parâmetros de usinagem sobre rugosidade, sabe-se que o avanço e o raio de ponta da ferramenta são os parâmetros mais influentes, de forma que a rugosidade aumenta com o quadrado do avanço e é inversamente proporcional ao raio de ponta 0 . No entanto, a diminuição da rugosidade com a redução do avanço não é contínua, existindo, portanto, um valor mínimo de avanço que deve ser utilizado. Esse comportamento acontece porque para valores muito pequenos de avanço não se atinge a espessura mínima de corte para a formação do cavaco, fazendo com que ocorra sulcamento e a formação de fluxo lateral de material que prejudica a rugosidade 0. DAS et al. 0 avaliaram a influência dos parâmetros de torneamento do aço AISI 4140 e concluíram que o aumento da rugosidade com o avanço também recebe a contribuição da elevação da força de usinagem, que provoca a elevação da força de atrito e da deformação plástica. Já em relação ao raio de ponta, MEDDOUR et al. 0 afirmam que com o aumento do raio de ponta da ferramenta, os picos de rugosidade são esmagados, diminuindo a rugosidade da superfície usinada. Porém, um raio de ponta excessivamente elevado pode levar a uma intensificação das vibrações, prejudicando a formação do cavaco e aumentando a rugosidade 0 .

Outro parâmetro considerado influente sobre a rugosidade é a velocidade de corte [6,7]; esta influência ocorre provavelmente devido à temperatura. Além de reduzir a possibilidade de surgimento da aresta postiça de corte 0 , o aumento inicial da velocidade de corte eleva a temperatura e tende a diminuir a resistência ao cisalhamento do material. Segundo MEDDOUR et al. 0 , um aumento na velocidade de corte (de 120 para $244 \mathrm{~m} / \mathrm{min}$ ) no torneamento do aço AISI 4140 foi capaz de diminuir a influência da variação do avanço sobre a rugosidade, (as marcas de avanço se mantiveram, porém, a amplitude dos picos foi diminuída). Os autores destacam ainda que o aumento da velocidade de corte, além de ser benéfico para a redução da rugosidade, favorece o aumento da produtividade. Um aumento excessivo, no entanto, pode levar ao desgaste acentuado da ferramenta de corte e prejudicar o acabamento da peça [4,5]. Além disso, a elevação da velocidade de corte eleva o nível de vibrações, o que também prejudica a rugosidade [5]. Segundo BHAT e PATIBANDLA 0, superfícies rugosas prejudicam a vida em fadiga porque proporcionam um maior número de regiões de início de trincas.

Tensões residuais são aquelas que estão presentes em um material quando este está em equilíbrio com o seu entorno, ou seja, na ausência de forças externas ou gradientes térmicos. A origem dessas tensões pode ser mecânica, térmica ou química. As tensões de origem mecânica são normalmente o resultado de deformações plásticas não uniformes causadas pelos processos de fabricação 0 . Segundo KANDIL et al. 0 , as técnicas de medição de tensões residuais normalmente medem deformações em vez de tensões. As tensões são deduzidas usando as propriedades dos materiais, tais como módulo de elasticidade e coeficiente de Poisson. Um dos métodos de medição das tensões é o método do furo cego incremental. Neste método, um furo é usinado na superfície da peça enquanto as deformações são medidas. Dentre os métodos de medição da deformação, as rosetas 0 formadas por três extensômetros permitem a medição de tensões em duas direções de um plano 0.

Segundo JAVIDI et al. 0, as tensões residuais dependem do material a ser trabalhado e também dos parâmetros utilizados. RECH e MOISAN 0 destacam ainda a importância da geometria e do material da ferramenta. Quando o processo é mecanicamente dominante, as tensões residuais tendem a ser compressivas e quando é termicamente dominante tendem a ser de tração 0 , portanto, o estado final das tensões depende da predominância de um fenômeno em relação ao outro 0 . A velocidade de corte é capaz de influenciar as tensões residuais superficiais induzidas na peça $[4,10]$, novamente devido à sua influência sobre a temperatura de corte. Já o material e o desgaste da ferramenta provocam variações nas condições de atrito entre a ferramenta e a peça modificando a geração de calor. Em geral, o desgaste da ferramenta aumenta o atrito entre sua superfície de folga e a superfície da peça recém gerada e, consequentemente, o efeito térmico, podendo induzir tensões de tração $[4,6]$. O material ou revestimento da ferramenta pode ou não ser benéfico dependendo dos materiais envolvidos 0 . Testes feitos por RAMI et al.0 mostraram que as tensões residuais induzidas no torneamento do aço AISI 4140 foram sempre de tração na superfície, passando para valores compressivos a partir de uma certa profundidade. Os autores afirmam que esse perfil de tensões é gerado pela ação conjunta da temperatura e das forças durante a operação de torneamento.

Testes de fadiga por flexão rotativa em amostras torneadas foram feitos por JAVIDI $e t$ al. 0 e YAO et al. 0 e os resultados indicaram que as amostras de aço AISI 43400 torneadas com rugosidades mais elevadas nem sempre apresentaram vida em fadiga mais curta. Os autores explicaram esse comportamento pela maior 
influência das tensões residuais do que da rugosidade sobre a vida em fadiga. Já os testes em uma liga de titânio 0 mostraram que nem sempre as tensões residuais dominam o comportamento. Nesse caso, a variação de rugosidade foi de cerca de três vezes de uma condição para outra. A condição de menor rugosidade foi a que propiciou menor intensidade de tensões compressivas, mas mesmo assim proporcionou vida em fadiga mais longa. Esse comportamento indica que a influência de cada uma das características depende também da amplitude e da faixa de variação entre um caso e outro.

Com base nos trabalhos relatados, é perceptível a influência dos parâmetros de usinagem no comportamento do material. Assim, o objetivo deste trabalho é avaliar a influência dos parâmetros de torneamento sobre a rugosidade, tensões residuais e vida em fadiga do aço ABNT 4140 temperado. Mais especificamente, serão avaliados os seguintes parâmetros: velocidade de corte, avanço e profundidade de usinagem.

\section{MATERIAIS E MÉTODOS}

O aço ABNT 4140 foi adquirido na forma de barras cilíndricas com diâmetro de 15,875 mm para produção dos corpos de prova aos ensaios de torneamento e fadiga, e de 50,8 $\mathrm{mm}$ para a medição da tensão residual do material. As barras destinadas ao torneamento dos corpos de prova de fadiga passaram por uma operação de desbaste utilizando velocidade de corte de $40 \mathrm{~m} / \mathrm{min}$, avanço de $0,2 \mathrm{~mm} / \mathrm{rev}$ e profundidade de usinagem de $1,0875 \mathrm{~mm}$. Para a região com variação de diâmetro foi utilizado um avanço de $0,03 \mathrm{~mm} / \mathrm{rev}$ para evitar esforços excessivos devido à variação de geometria. Em seguida, todas as amostras foram tratadas termicamente por têmpera $\left(870{ }^{\circ} \mathrm{C}\right.$ por 60 min seguido de resfriamento em óleo mineral a $90{ }^{\circ} \mathrm{C}$ por $\left.30 \mathrm{~s}\right)$ seguida de revenimento $\left(400{ }^{\circ} \mathrm{C}\right.$ por $120 \mathrm{~min}$ ). A dureza média adquirida do material termicamente tratado foi de $40 \pm 2$ HRC. Como a realização dos tratamentos térmicos foi posterior ao desbaste, os efeitos desta etapa de usinagem foram neutralizados. Os ensaios de torneamento de acabamento foram realizados em um torno comandado numericamente (potência de $5,5 \mathrm{~kW}$ e rotação máxima de $3500 \mathrm{rpm}$ ) com a utilização de insertos Mit-

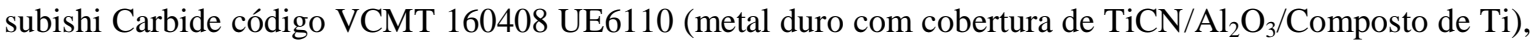
montados em um suporte código ISO SVVCN 2020-K16N do mesmo fabricante. A geometria e dimensão final dos copos de prova foram adequadas para o ensaio de fadiga, conforme a Figura 1.
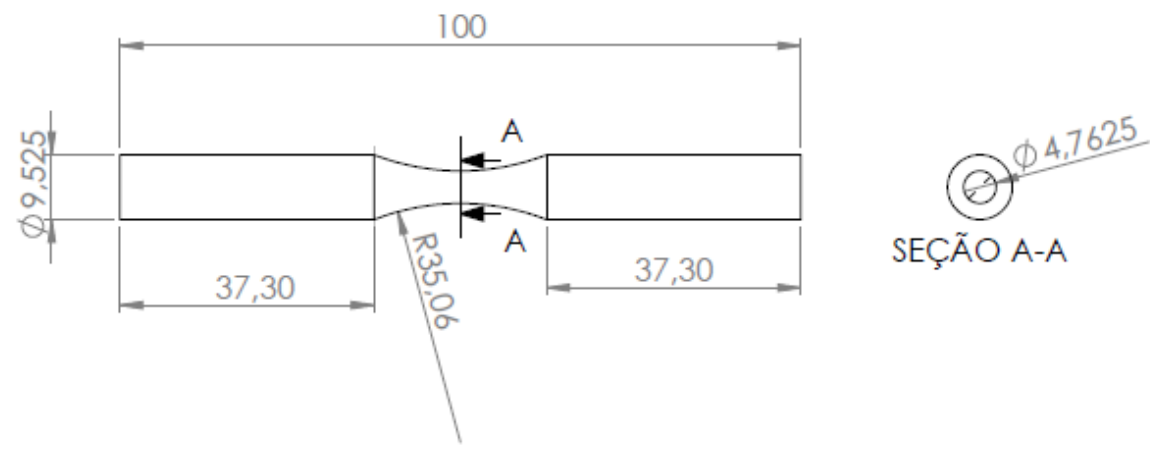

Figura 1: Desenho do corpo de prova.

A variação dos parâmetros do torneamento foi definida por um planejamento experimental $2^{\mathrm{k}}$ com três fatores (velocidade de corte, avanço e profundidade de usinagem) e utilizando duas réplicas, do qual totalizaram 24 ensaios. A Tabela 1 apresenta os níveis utilizados para cada fator.

Tabela 1: Fatores e níveis utilizados no torneamento dos corpos de prova.

\begin{tabular}{l|l|l}
\hline \multirow{2}{*}{ FATORES } & \multicolumn{2}{l}{ NíVEIS } \\
\cline { 2 - 3 } & - & + \\
\hline Velocidade de corte $\mathrm{v}_{\mathrm{c}}(\mathrm{m} / \mathrm{min})$ & 30 & 45 \\
\hline Avanço $\mathrm{f}(\mathrm{mm} / \mathrm{rev})$ & 0,2 & 0,3 \\
\hline Profundidade de usinagem $\mathrm{a}_{\mathrm{p}}(\mathrm{mm})$ & 0,5 & 1,0 \\
\hline
\end{tabular}

A tensão residual foi avaliada pelo método do furo cego incremental pelo equipamento RS-200 Milling Guide da Vishay Precision Group. Esse equipamento possui uma turbina de alta rotação utilizada para a realiza- 
ção de furos empregando fresas JET FG 38 (diâmetro de 1,6 mm) e um micrômetro acoplado. Antes dos ensaios, todas as amostras foram limpas e atacadas com nital para fixação das rosetas extensométricas EA-06-062RE120 (Excel, São Paulo) na superfície de interesse, utilizando o adesivo Permabond 793. Os terminais de cada roseta extensométrica foram então conectados ao equipamento responsável por registrar os sinais relativos às deformações sofridas pelos extensômetros durante a realização dos furos no material. Os valores de deformação são registrados em incrementos de $0,05 \mathrm{~mm}$ de profundidade do furo para a formação do perfil de tensão a partir da superfície do corpo de prova. Os valores de deformação foram tratados no software H-drill, o qual foi alimentado com informações sobre as propriedades do material em estudo, para complementar o cálculo da tensão residual. Como a medição de tensões residuais pelo método do furo cego incremental requer a fixação da roseta extensométrica sobre uma superfície plana, a medição de tensões residuais foi realizada nos corpos de prova com diâmetro de $50,8 \mathrm{~mm}$, os quais foram tratados termicamente da mesma forma que os corpos de prova de fadiga e depois faceados sob condições de corte idênticas às apresentadas na Tabela 1. Para tanto, a operação de faceamento foi executada utilizando programação com velocidade de corte constante controlada pelo torno, isto é, a rotação dos corpos de prova foi corrigida (elevada) automaticamente pelo comando numérico à medida que a ferramenta de corte avançava até o centro da peça (respeitando a rotação máxima do torno), de forma que a velocidade de corte (tangencial) se mantivesse constante. Além disso as rosetas foram fixadas em uma região onde a rotação do torno permitia atingir a velocidade de corte desejada. Desta forma, a medição de tensões residual foi efetuada na face plana destes corpos de prova.

Após os ensaios de torneamento, realizou-se a medição de rugosidade de todas as peças com a utilização do perfilômetro (Hommelwerke modelo Hommel Etamic T8000). Esse equipamento permite a medição da rugosidade superficial sem que a variação de diâmetro do corpo de prova venha a interferir nos resultados, a qual é possível devido à existência da função de nivelamento do software de análise das medições (Hommelmap Expert 6.2). Os parâmetros de amplitude da rugosidade avaliados foram Rq, Rt, Rv, Rp, Rz, Rvk, Rk e Rpk.

Os testes de fadiga foram realizados em uma máquina de fadiga por flexão rotativa (Fatigue Dynamics modelo RBF - 200) com rotação de 5000 rpm e aplicando-se uma tensão de 590,5 MPa, que corresponde a $50 \%$ da tensão de escoamento do material. Foram feitos três testes de fadiga para cada condição de corte apresentada na Tabela 1, sendo registrado o número de ciclos até a ruptura. A tensão foi escolhida baseada em ensaios preliminares de tração do material. Os ensaios de tração foram realizados em uma máquina universal de ensaios Shimadzu modelo AG-IS mostrada na Figura 2, com célula de carga de $100 \mathrm{kN}$ modelo SLFL, com o objetivo de determinar o limite de escoamento a $0,2 \%$ de deformação. As dimensões destes corpos de prova, conforme apresentadas na Figura 3, foram baseadas na norma NBR 6152:2002 0 e determinadas em função da capacidade da célula de carga da máquina universal. As curvas tensão x deformação para o material em questão estão na Figura 4.

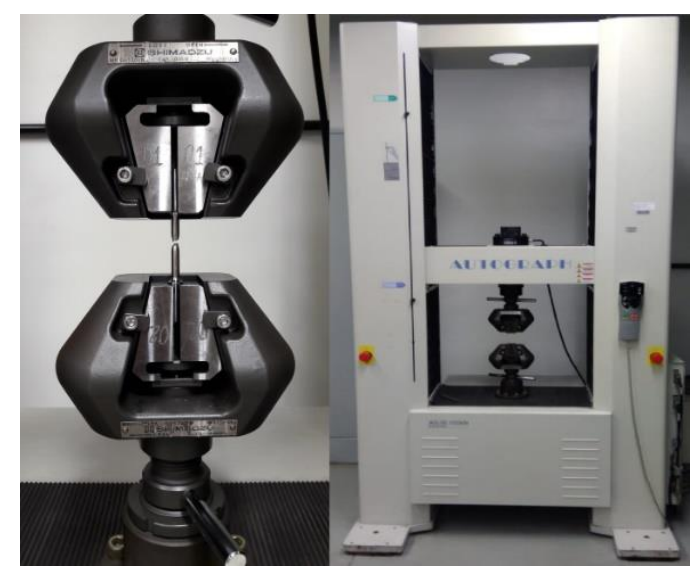

Figura 2: Máquina de tração utilizada. 


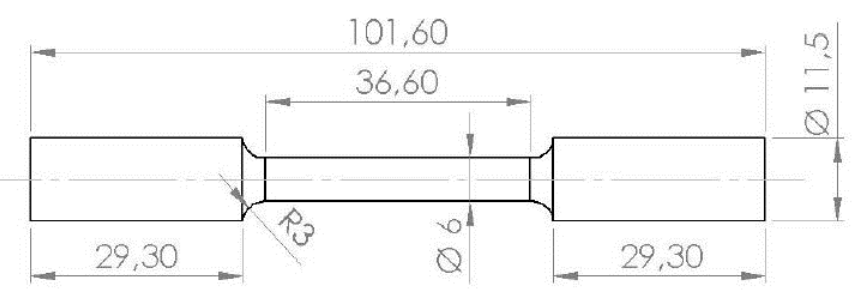

Figura 3: Desenho do corpo de prova para tração.

\section{RESULTADOS E DISCUSSÃO}

Nesta seção são apresentados e discutidos os resultados obtidos neste trabalho.

A Figura 4 apresenta as curvas tensão x deformação de duas amostras de aço ABNT 4140. O valor médio do limite de resistência é $1371 \mathrm{MPa}$ e da tensão de escoamento é $1181 \mathrm{MPa}$ (para 0,2\% de deformação), consequentemente, os testes de fadiga foram realizados aplicando uma tensão correspondente à metade da tensão de escoamento (590,5 MPa).

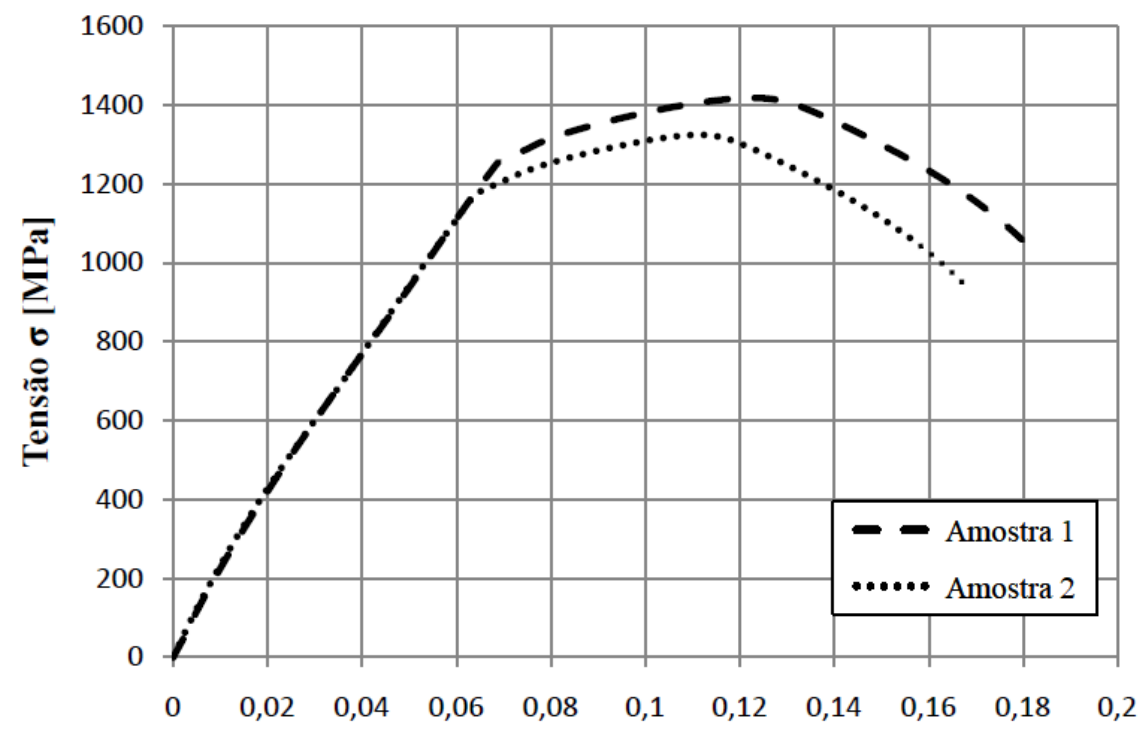

Deformação $\epsilon[\mathrm{mm} / \mathrm{mm}]$

Figura 4: Curvas tensão x deformação verdadeiras.

\subsection{Rugosidade}

Os parâmetros de amplitude Rq, Rt, Rv, Rp, Rz, Rvk, Rk e Rpk são apresentados conforme suas análises em relação as condições do torneamento. Os resultados de desvio médio quadrático (Rq) e altura total do perfil (Rt) podem ser observados na Figura 5. As rugosidades Rq e Rt apresentam comportamento semelhante, sendo também percebido que o avanço mais elevado $(0,3 \mathrm{~mm} / \mathrm{rev})$ tende a aumentar o valor da rugosidade. Além da influência do avanço sobre a distância entre picos e vales do perfil gerado, DAS et al. 0 afirmam que esse comportamento recebe a contribuição adicional do aumento da força de avanço, que resulta em vibração e pode aumentar a rugosidade superficial. 


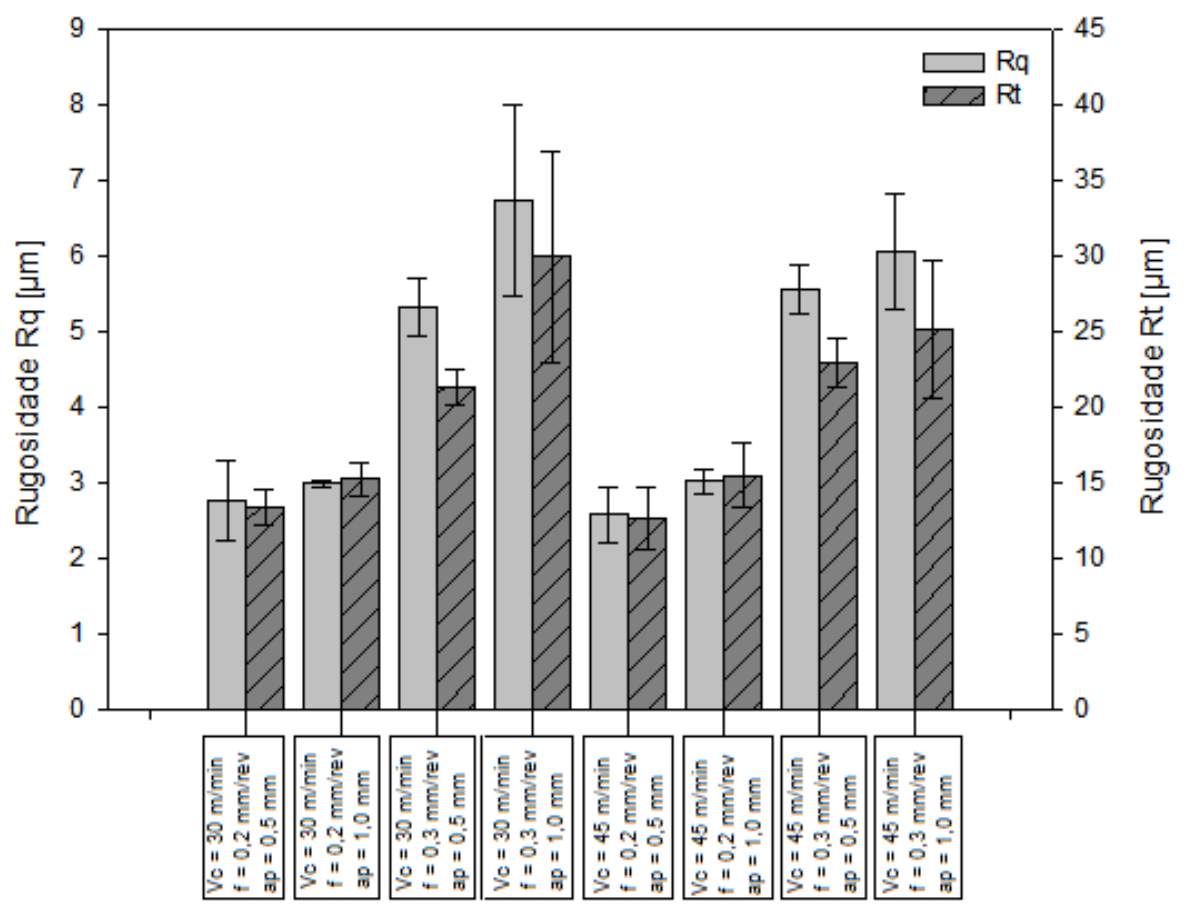

Condições de torneamento

Figura 5: Variação do desvio médio quadrático (Rq) e da altura total do perfil (Rt) com os parâmetros de corte.

A profundidade de usinagem também demonstra interferir em Rq, porém em menor intensidade do que o avanço. Novamente a elevação das forças de usinagem pode explicar esta variação da rugosidade. Com relação à velocidade de corte nota-se uma ligeira queda na rugosidade, principalmente associada ao menor valor de avanço, no entanto, esta variação não excede a dispersão dos dados.

A influência dos parâmetros de corte sobre a altura máxima do perfil (Rz), a qual é obtida pela soma da altura máxima dos picos (Rp) com a profundidade máxima dos vales (Rv), é apresentada na Figura 6. Do mesmo modo que Rq, o parâmetro Rz mostra baixa correlação com o aumento da $\mathrm{a}_{\mathrm{p}}$, ou seja, a profundidade mais alta pode provocar o aumento das componentes da força de torneamento, elevando a valores de rugosidade. Conforme relatado por DAS et al. 0, o avanço mostra-se o parâmetro de torneamento mais influente. Já a velocidade de corte mais alta $(45 \mathrm{~m} / \mathrm{min}$ ) parece promover a redução de Rp e Rv, particularmente quando é empregado avanço mais elevado ( $\mathrm{f}=0,3 \mathrm{~mm} / \mathrm{rev})$. O aumento da temperatura com a velocidade de corte pode ter reduzido a resistência ao cisalhamento do material, propiciando uma redução da rugosidade.

O perfil de rugosidade também foi avaliado por meio da curva razão de material representada na Figura 7. Esta mostra a razão entre o material e o ambiente externo e é iniciada no primeiro contato com um pico do perfil e indica a modificação desta razão com a profundidade à medida que se aproxima do interior do material. Dentre os diversos parâmetros obtidos a partir desta curva, os mais estudados são: Rpk (altura de pico reduzida), Rk (profundidade da rugosidade central) e Rvk (profundidade de vales reduzida), que são relacionados, respectivamente, à altura dos picos, região central do perfil e a profundidade dos vales. 


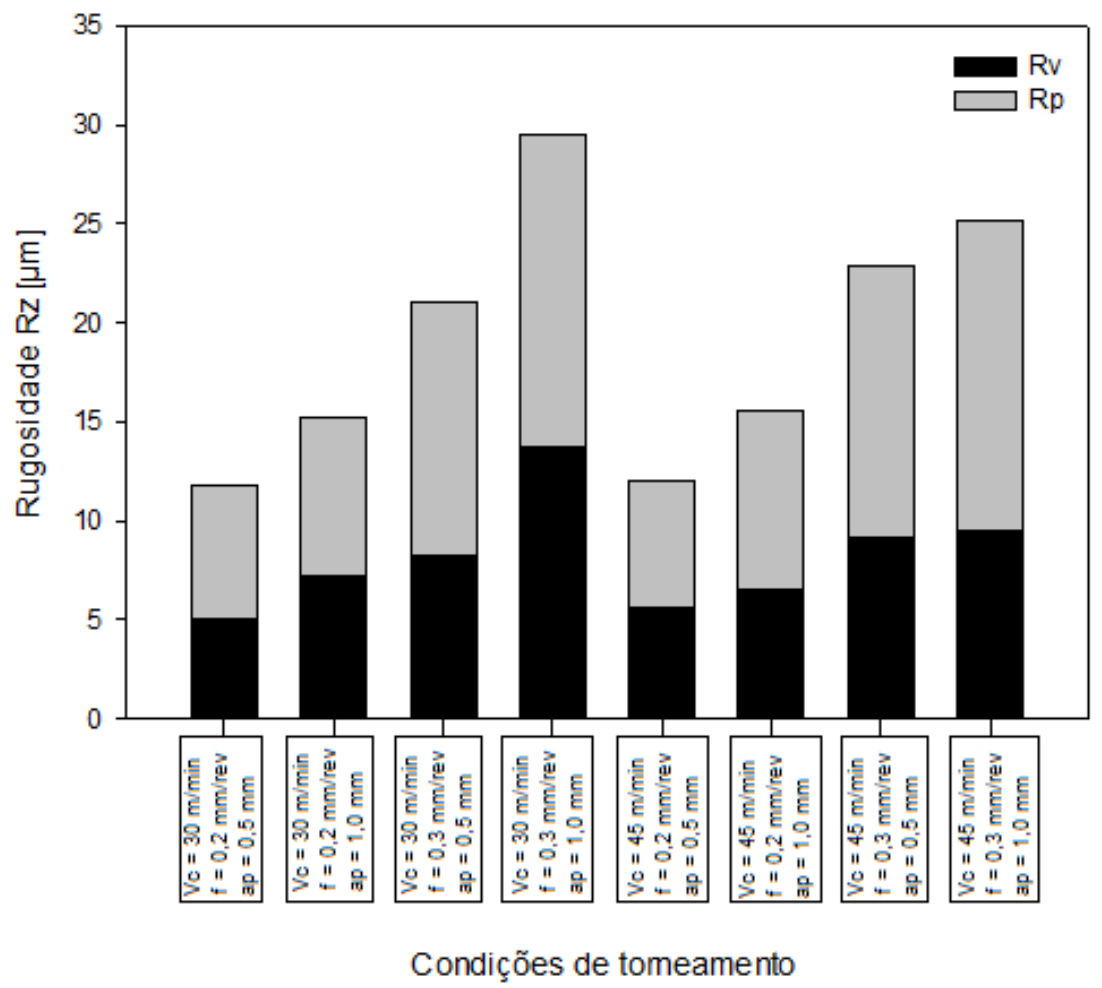

Figura 6: Variação da altura máxima do perfil (Rz), altura máxima dos picos (Rp) e profundidade máxima dos vales (Rv) com os parâmetros de corte

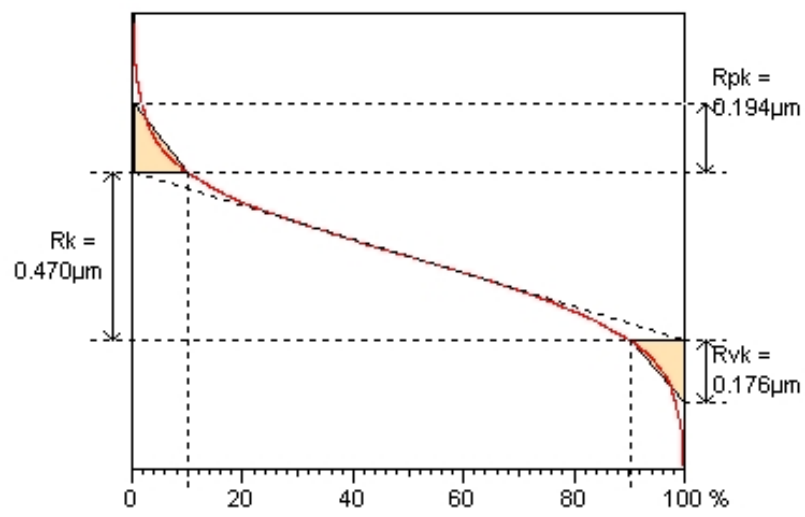

Figura 7: Exemplo de curva razão de material.

Com o auxílio das curvas geradas para cada perfil foi possível obter o gráfico mostrado na Figura 8 , que relaciona os parâmetros Rpk, Rk e Rvk às condições de torneamento. Novamente, pode-se perceber que o avanço é o fator que possui a maior influência sobre os parâmetros Rpk e Rk, que tenderam a aumentar com a elevação do avanço. Além disso, a razão entre os parâmetros, que indica a proporção entre picos e vales presentes, se altera com a modificação dos parâmetros de entrada. O parâmetro Rpk, que é relacionado aos picos do perfil, possui uma nítida tendência de variação apenas com o avanço, ao passo que as variações de velocidade de corte e profundidade de usinagem parecem não interferir. Um comportamento diferente foi observado para o parâmetro associado à região central do perfil (Rk), que além de ser influenciado pelo avanço ainda parece sofrer a influência da profundidade de usinagem e da velocidade. Para velocidades de corte mais baixas, a variação da profundidade de usinagem tem uma capacidade maior de afetar a amplitude de $\mathrm{Rk}$, comportamento que não ocorre para o valor mais elevado de velocidade de corte, possivelmente devido à temperatura mais elevada do processo. 


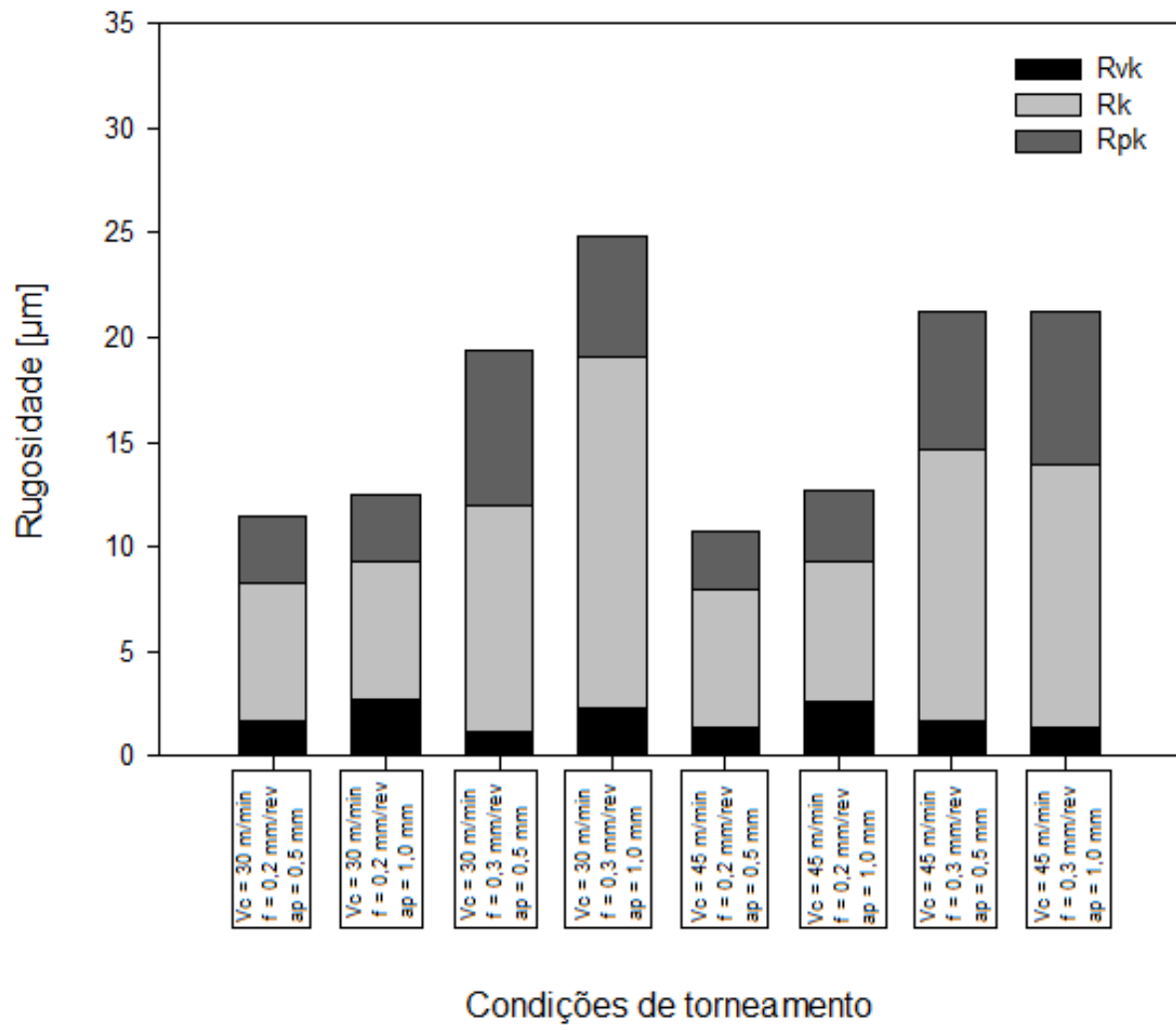

Figura 8: Variação da profundidade de vales reduzida (Rvk), profundidade da rugosidade central (Rk) e altura de pico reduzida (Rpk) com os parâmetros de corte.

Já o parâmetro Rpk é influenciado tanto pela profundidade de usinagem quanto pelo avanço. Com a elevação do avanço a maior parte do perfil é formada por picos, diminuindo a contribuição dos vales. Já para profundidades de usinagem mais elevadas, o parâmetro do vale (Rvk) é, na maioria das vezes, de maior amplitude. Já a velocidade de corte mais elevada parece influenciar apenas quando combinada com o avanço mais elevado, já que nessa condição a variação provocada pela profundidade de usinagem é eliminada.

Para todos os parâmetros de saída mostrados anteriormente, foi feita uma análise de variância para avaliar quais parâmetros de entrada e quais interações são significativas $(\checkmark)$. Foi considerado um nível de significância de $5 \%$. Um resumo dessa análise pode ser observado na Tabela 2.

Tabela 2: Resumo da influência dos fatores estudados sobre os parâmetros de rugosidade.

\begin{tabular}{l|l|l|l|l|l|l|l|l}
\hline & \multicolumn{7}{|c}{ PARÂMETROS } \\
\hline FATOR/INTERAÇÃO & Rq & Rt & Rz & Rp & Rv & Rpk & Rk & Rvk \\
\hline $\mathrm{V}_{\mathrm{c}}$ & & & & & & & & \\
\hline $\mathrm{f}$ & $\checkmark$ & $\checkmark$ & $\checkmark$ & $\checkmark$ & $\checkmark$ & $\checkmark$ & $\checkmark$ & \\
\hline $\mathrm{a}_{\mathrm{p}}$ & $\checkmark$ & $\checkmark$ & $\checkmark$ & $\checkmark$ & $\checkmark$ & & & $\checkmark$ \\
\hline $\mathrm{V}_{\mathrm{c}} * \mathrm{f}$ & & & & & & & & \\
\hline $\mathrm{V}_{\mathrm{c}} * \mathrm{a}_{\mathrm{p}}$ & & & & & & $\checkmark$ & $\checkmark$ & \\
\hline $\mathrm{f} * \mathrm{a}_{\mathrm{p}}$ & & & & & & & & \\
\hline $\mathrm{V}_{\mathrm{c}} * \mathrm{f} * \mathrm{a}_{\mathrm{p}}$ & & & & & & & & \\
\hline
\end{tabular}

O comportamento observado para os parâmetros de rugosidade em função dos fatores estudados está de acordo com a literatura. Segundo MEDDOUR et al. 0, a rugosidade tende a aumentar com o avanço. Esse comportamento foi percebido para a maior parte dos fatores analisados, já que o avanço foi estatisticamente influente e levou a um aumento dos parâmetros de rugosidade. Além do avanço, a profundidade de usinagem também afetou negativamente a rugosidade, porém em menor intensidade do que o avanço. Esse comportamento pode ter ocorrido devido à elevação dos esforços de corte com a profundidade de usinagem. 


\subsection{Tensões residuais}

Os gráficos com os valores de tensão residual em função da profundidade abaixo da superfície para as diferentes condições de torneamento são apresentados nas Figuras 9 e 10 para velocidades de corte de 30 e 45 $\mathrm{m} / \mathrm{min}$, respectivamente. As direções X e Y de medição das tensões são paralela e perpendicular à direção do avanço, respectivamente.
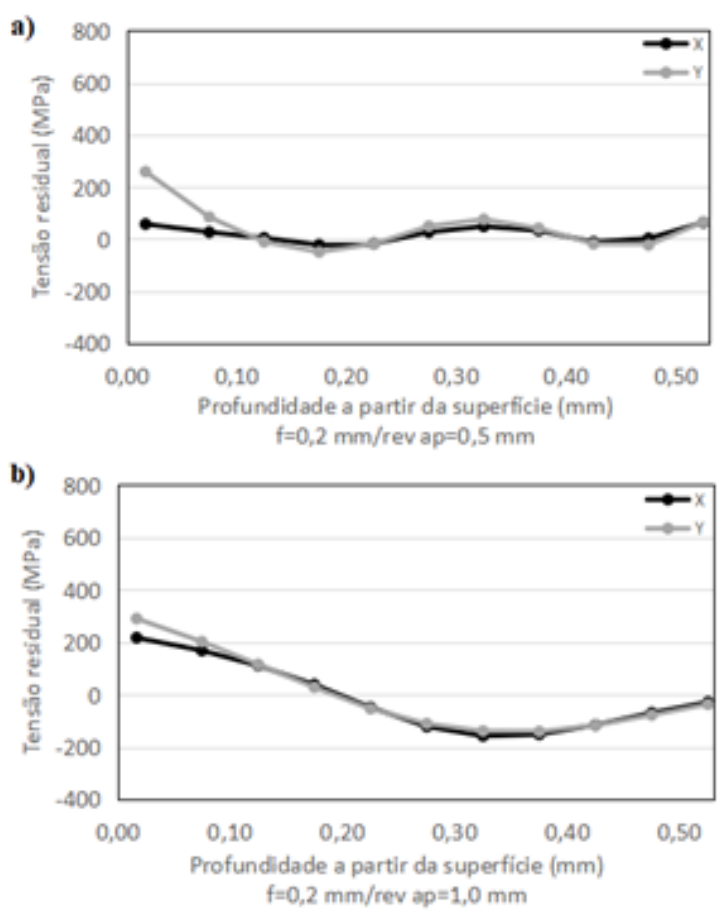

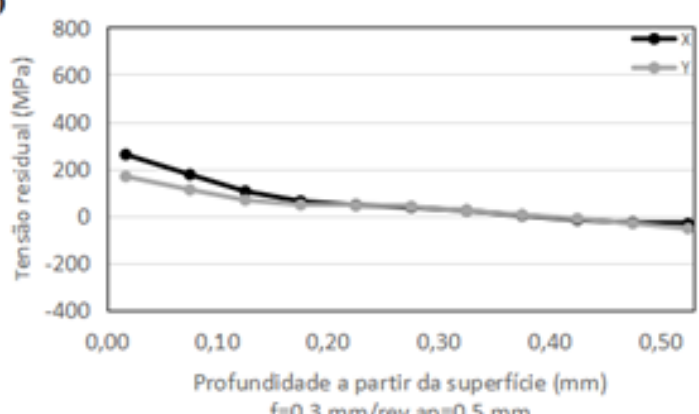

$f=0,3 \mathrm{~mm} / \mathrm{rev}$ ap $=0,5 \mathrm{~mm}$

Figura 9: Tensões residuais para as amostras torneadas sob velocidade de corte de $30 \mathrm{~m} / \mathrm{min}$.
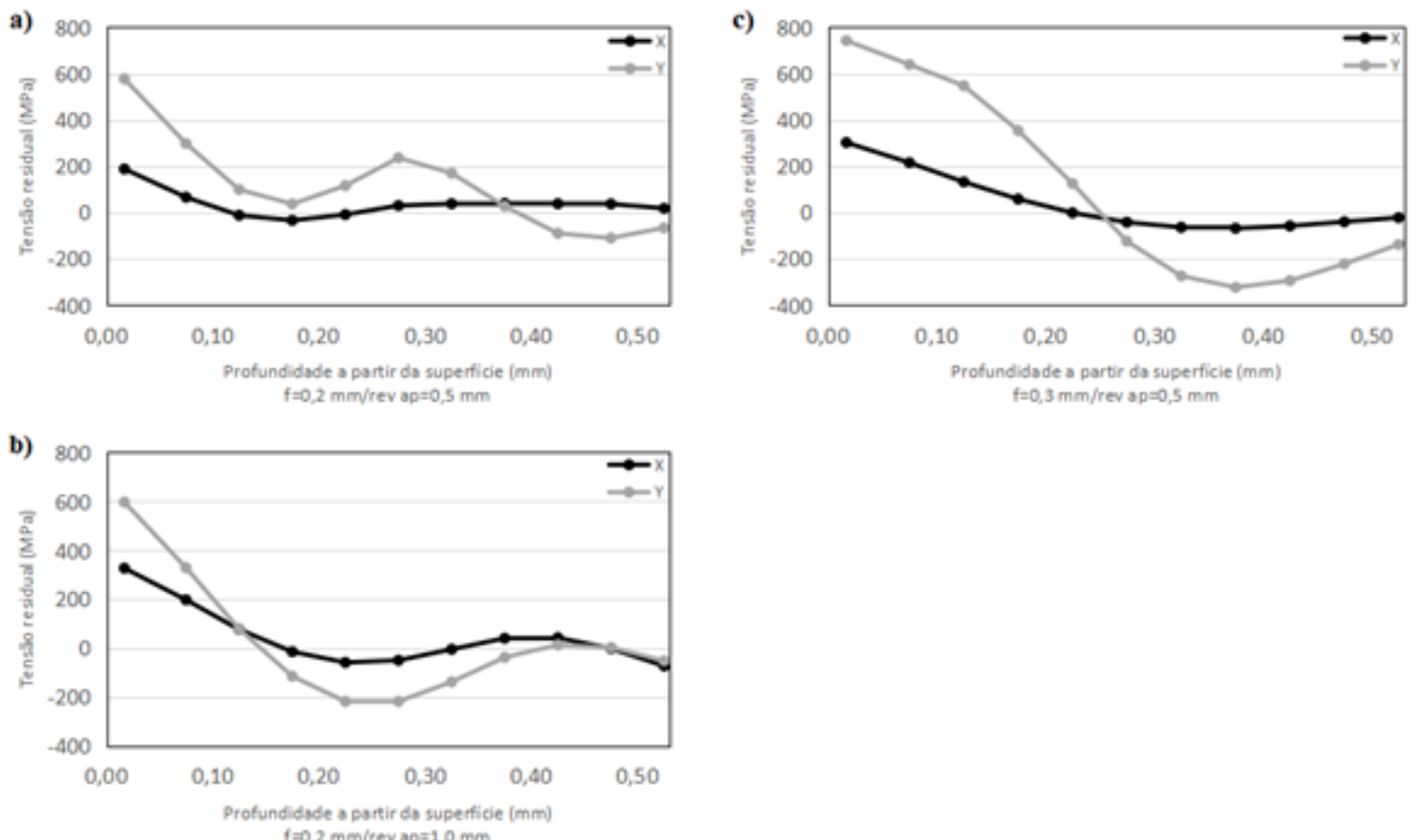

$=0.2$ mmitex ap $=1.0 \mathrm{~mm}$

Figura 10: Tensões residuais para as amostras torneadas sob velocidade de corte de $45 \mathrm{~m} / \mathrm{min}$.

Ao observar as Figuras 9 e 10, pode-se perceber que em todas as combinações de parâmetros a tensão residual superficial apresenta caráter de tração próximo à superfície do material. Além disso, para a velocida- 
de de corte mais alta, as tensões residuais apresentam maior intensidade. Os valores de avanço e profundidade de usinagem também são capazes de alterar o perfil de tensões. Para o avanço mais baixo, o aumento da profundidade de usinagem tende a aumentar a intensidade das tensões compressivas, comportamento que não acontece para os avanços mais elevados. Este comportamento foi o mesmo observado por RAMI et al. 0 na usinagem do aço AISI 4140, onde tensões residuais de tração foram detectadas na superfície, podendo se converter em compressivas a partir de uma certa profundidade. Além de apresentar esse perfil, as tensões residuais tenderam a apresentar intensidade mais elevada para a velocidade de corte mais alta. Segundo JACOBUS et al. 0, quando as tensões são termicamente dominantes elas tendem a ser de tração. Comparando as Figuras 9 e 10 observa-se que o aumento da velocidade de corte provavelmente acentuou o caráter térmico do processo, levando a tensões residuais de tração mais intensas. Com relação aos demais parâmetros, tem-se que a redução do avanço e o aumento da profundidade de usinagem tendem a aumentar a intensidade das tensões compressivas abaixo da superfície, fato que pode ser vinculado aos maiores esforços de usinagem para estas condições. Já para o avanço mais elevado, esse comportamento não foi percebido, provavelmente devido ao aumento do efeito térmico.

\subsection{Vida em fadiga}

A Figura 11 apresenta os valores médios e os desvios padrões para a vida em fadiga de corpos de prova produzidos sob diferentes condições de torneamento. O comportamento em fadiga foi diferente para cada velocidade de corte: para a velocidade de corte mais baixa $(30 \mathrm{~m} / \mathrm{min})$, a elevação do avanço promoveu um aumento da vida em fadiga, já para a velocidade mais alta $(45 \mathrm{~m} / \mathrm{min})$ a elevação de avanço provocou uma diminuição. Outro comportamento que pode ser observado neste gráfico é a variação do número de ciclos até a falha com o aumento da profundidade de usinagem para cada avanço: com o avanço baixo $(0,2 \mathrm{~mm} / \mathrm{rev})$, o aumento da profundidade de usinagem promoveu vida em fadiga mais longa, comportamento que não foi notado para o avanço mais elevado $(0,3 \mathrm{~mm} / \mathrm{rev})$. O aumento do avanço pode ter contribuído para a indução de tensões residuais de tração devido à intensificação do efeito térmico do processo, sobrepondo a influência mecânica gerada pelo aumento de forças com a profundidade de usinagem.

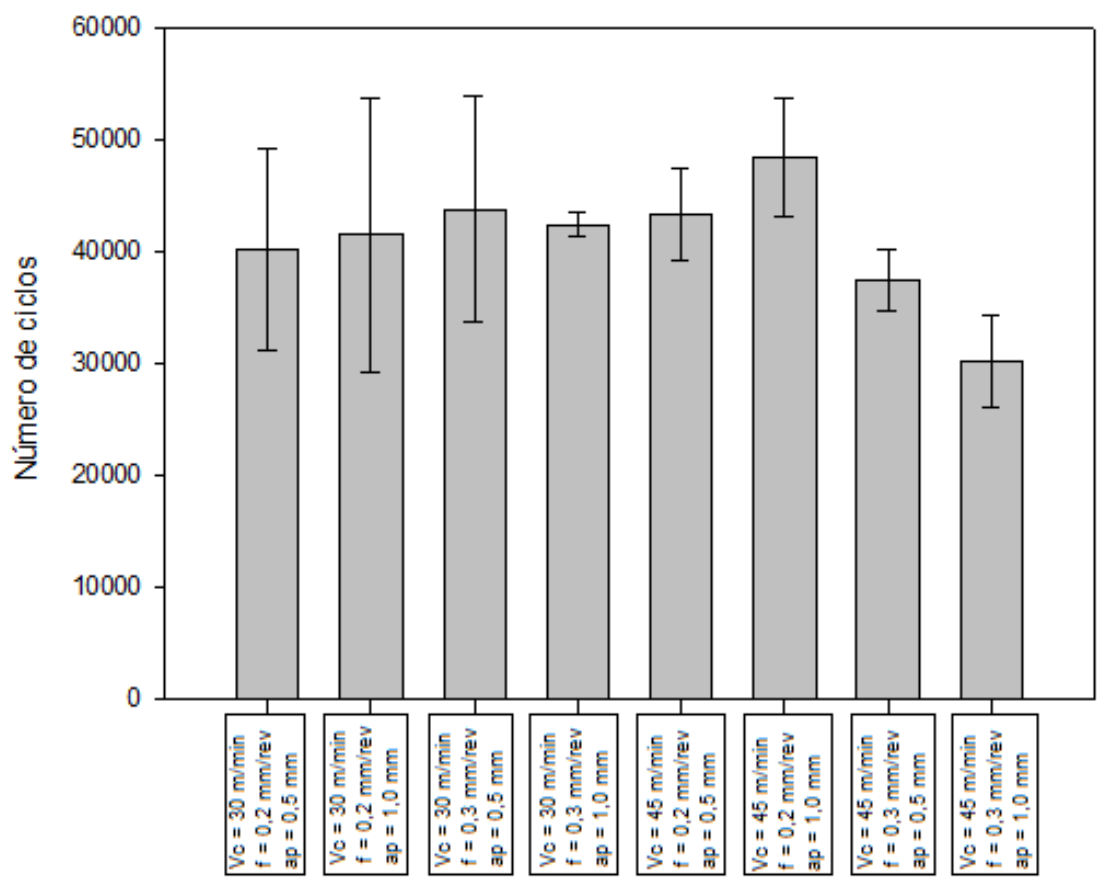

Condições de tomeamerto

Figura 11: Influência das condições de torneamento sobre a vida em fadiga.

Além disso, percebe-se que as condições nas quais se combinam valores mais altos de rugosidade e tensões residuais de tração de maior intensidade (velocidade de corte de $45 \mathrm{~m} / \mathrm{min}$ e avanço de $0,3 \mathrm{~mm} / \mathrm{rev}$ ), proporcionam vidas em fadiga mais curtas. Porém, essas características isoladas não definem o melhor com- 
portamento da vida em fadiga do material, uma vez que tensões residuais superficiais de tração de maior intensidade combinadas com menor rugosidade proporcionaram a vida em fadiga mais longa. O comportamento das tensões residuais realmente interfere na resistência a fadiga do material, já que o mesmo comportamento combinado da profundidade com o avanço que modificou o perfil das tensões residuais também afetou a vida em fadiga. Para o avanço mais baixo, o aumento da profundidade de usinagem tendeu a aumentar a vida em fadiga; já para o avanço mais elevado o comportamento foi oposto, sendo mais facilmente percebido para as amostras torneadas com a velocidade de $45 \mathrm{~m} / \mathrm{min}$ (que receberam a contribuição do efeito térmico associado ao avanço e à velocidade de corte elevados).

\section{CONCLUSÕES}

Após a produção de corpos de prova em aço ABNT 4140 temperado e revenido (40 HRC) por meio da operação de torneamento sob condições distintas de corte e a avaliação de diversos parâmetros de rugosidade da superfície usinada, tensão residual induzida no material e vida em fadiga, conclui-se que:

- Com relação aos parâmetros de rugosidade, o avanço foi o principal responsável pela variação dos parâmetros, que tendem a ser mais elevados para o valor mais alto de avanço. A rugosidade também foi afetada pela profundidade de usinagem (a profundidade de usinagem mais elevada levou a valores mais altos de rugosidade), porém em influência menor do que a do avanço.

- A tensão residual foi afetada por todos os parâmetros de usinagem utilizados: a velocidade de corte influenciou a intensidade das tensões residuais superficiais (o aumento da velocidade de corte induziu tensões residuais de tração de maior intensidade). Já em relação ao avanço e à profundidade de usinagem, ocorreu um comportamento combinado, isto é, para o avanço baixo, o aumento da profundidade de usinagem elevou a intensidade das tensões compressivas na subsuperfície. Já para o avanço mais elevado, o aumento da profundidade de usinagem não gerou um aumento das tensões compressivas.

- A vida em fadiga foi influenciada de forma combinada pela rugosidade e tensões residuais. Valores mais elevados de rugosidade combinados com tensões residuais mais intensas de tração propiciaram vida em fadiga mais curta, provavelmente devido a uma maior facilidade de nucleação e propagação das trincas. Os fatores que proporcionaram essas condições indesejáveis foram velocidade de corte e avanço mais elevados, independentemente da profundidade de usinagem utilizada. A vida em fadiga mais longa foi obtida com a velocidade de corte e profundidade de usinagem mais altas aliadas ao avanço mais baixo.

\section{AGRADECIMENTOS}

O presente trabalho foi realizado com apoio da Coordenação de Aperfeiçoamento de Pessoal de Nível Superior - Brasil (CAPES) - Código de Financiamento 001, da Fundação de Amparo à Pesquisa do Estado de Minas Gerais - FAPEMIG (projeto TEC-APQ-00241-11) e do Conselho Nacional de Desenvolvimento Científico e Tecnológico - CNPq.

\section{BIBLIOGRAFIA}

[1] BHAT, S., PATIBANDLA, R., Metal Fatigue and Basic Theoretical Models: A Review, Alloy Steel Properties and Use, Dr. Eduardo Valencia Morales (Ed.), ISBN: 978-953- 307-484-9, InTech, pp. $203-246$. Dez. 2011.

[2] JAVIDI, A., RIEGER, U., EICHLSEDER, W., "The effect of machining on the surface integrity and fatigue life", International Journal of Fatigue, v. 30, pp. 2050-2055, 2008. Disponível em: <https://linkinghub-elsevier-com.ez27.periodicos.capes.gov.br/retrieve/pii/S0142112308000133>

[3] MEDDOUR, I., YALLESE, M. A., BENSOUILAH, H., et al., "Prediction of surface roughness and cutting forces using RSM, ANN, and NSGA-II in finish turning of AISI 4140 hardened steel with mixed ceramic tool", The International Journal of Advanced Manufacturing Technology, v.97, pp.1931-1949, 2018. Disponível em: <https://link-springer-com.ez27.periodicos.capes.gov.br/article/10.1007/s00170-018-2026-6>

[4] RECH, J., MOISAN, A., "Surface integrity in finish hard turning of case-hardened steels", International Journal of Machine Tools \& Manufacture, v. 43, pp. 543-550, 2003. Disponível em: <https://wwwsciencedirect.ez27.periodicos.capes.gov.br/science/article/pii/S0890695502001414>

[5] DAS, S. R., DHUPAL, D., KUMAR, A. "Study of surface roughness and flank wear in hard turning of AISI 4140 steel with coated ceramic inserts", Journal of Mechanical Science and Technology, v.29, n.10, pp. 4329-4340, 2015. Disponível em: <https://link-springercom.ez27.periodicos.capes.gov.br/article/10.1007/s12206-015-0931-2> 
[6] BRANCO, F. K., DELIJAICOV, S., BORDINASSI, E. C., et al., "Surface Integrity Analisys in the Hard Turning of Cemented Steel AISI 4317", Materials Research, v. 21, n.5, Jul. 2018.

[7] YAO, C., LIN, J., WU, D., REN, J., "Surface integrity and fatigue behavior when turning $\gamma$-TiAl alloy with optimized PVD-coated carbide inserts", Chinese Journal of Aeronautics, v. 31, n. 4, pp. 826-836, 2018. Disponível em: <https://linkinghub-elsevier-

com.ez27.periodicos.capes.gov.br/retrieve/pii/S1000936117301413>

[8] KANDIL, F. A., LORD, J. D., FRY, A. T., et al., "A Review of Residual Stresss Measurement Methods A Guide to Technique Selection", NPL Report MATC(A) v.04, pp. 42, 2001.

[9] WITHERS, P. J., BHADESHIA, K. D. H., "Residual stress Part 1 - Measurement techniques", Materials Science and Technology. V. 17, pp. 355-365, 2001.

[10] JACOBUS, K., DEVOR, R. E., KAPOR, S. G., PEASCOE, R. A., "Predictive Model for the Full Biaxial Surface and Subsurface Residual Stress Profiles from Turning", Journal of Manufacturing Science and Engineering, v. 123, pp.537-546. 2001. Disponível em:

<http://manufacturingscience.asmedigitalcollection.asme.org/article.aspx?articleid=1484937>

[11] RAMI, A., KALLEL, A., SGHAIER, S., et al., "Residual stresses computation induced by turning of AISI 4140 steel using 3D simulation based on a mixed approach", International Journal of Advanced Manufacturing Technology, v.91, pp. 3833-3850, 2017. Disponível em: < https://link-springercom.ez27.periodicos.capes.gov.br/article/10.1007/s00170-017-0047-1>

[12] ASSOCIAÇÃO BRASILEIRA DE NORMAS TÉCNICAS. NBR 6152: "Materiais metálicos - Ensaio de tração à temperatura ambiente", Rio de Janeiro: ABNT, 2002.

\section{ORCID}

Augusto Moura Martins

Poliana Santos Souza

Anderson Júnior dos Santos

Alexandre Mendes Abrão

Berend Denkena

Bernd Breidenstein

Kolja Meyer
https://orcid.org/0000-0003-1456-087X

https://orcid.org/0000-0002-6864-0253

https://orcid.org/0000-0002-5285-3289

https://orcid.org/0000-0003-2015-4077

https://orcid.org/0000-0001-9763-6248

https://orcid.org/0000-0001-8899-9100

https://orcid.org/0000-0003-1384-5961 